\title{
Frost spreading and pattern formation on microstructured surfaces
}

\author{
Lukas Hauer $\odot,{ }^{1}$ William S. Y. Wong $₫{ }^{1}$ Azadeh Sharifi-Aghili, ${ }^{1}$ Lou Kondic $\odot,{ }^{2,}{ }^{*}$ and Doris Vollmer ${ }^{1, \dagger}$ \\ ${ }^{1}$ Physics at Interfaces, Max Planck Institute for Polymer Research, Ackermannweg 10, 55128 Mainz, Germany \\ ${ }^{2}$ Department of Mathematical Sciences and Center for Applied Mathematics and Statistics, \\ New Jersey Institute of Technology, \\ Newark, NJ 07102, USA
}

(Received 20 May 2021; accepted 31 August 2021; published 1 October 2021)

\begin{abstract}
Frost is found in nature as a symphony of nucleation and heat and mass transport, cascading from angstroms to several meters. Here, we use laser-induced fluorescence microscopy to investigate the pattern formation of frost growth in experiments which tune the mesoscopic length scale by using microstructured pillar arrays as a frost condenser surface. By controlling the degree of surface supercooling and the amount of condensate, different modes of frost patterning are uncovered, ranging from complete surface coverage to fractal-looking and limited-coverage structures of spiky appearance.
\end{abstract}

DOI: 10.1103/PhysRevE.104.044901

\section{INTRODUCTION}

Condensation frosting is the process of frost formation on partially wettable surfaces. Due to the high availability of water vapor in the atmosphere, condensation frosting is abundant in nature and technology. Understanding this process is not only fascinating for its complexity, but also highly important since frosting becomes detrimental in many technical applications. Condensation frosting starts with: (i) condensation of supercooled droplets [1-3], followed by (ii) the solidification of isolated droplets [4-6], and (iii) subsequent frost spreading, starting from a solidified droplet $[7,8]$. In step (iii), frost spreads by draining water from surrounding liquid droplets. Spreading evolves in one of two possible modes where the ratio of droplet diameter to interdroplet spacing is pivotal: If droplets are sufficiently large and distributed densely, frost propagates fast via connecting frost bridges. This spreading mode is characterized as diffusion (through air) followed by freezing of the connected droplets. The second mode is facilitated when individual droplets condense too sparsely. Droplets evaporate entirely before interdroplet frost bridges can connect them (i.e., dry zones form) [9]. In this second case, frost grows solely via diffusion without droplet freezing, similar to snowflakes in clouds [10]. Which of these two spreading modes applies is determined by isolated droplet-to-droplet interactions which are fully characterized in one dimension [11-13]. When droplets condense on surfaces, this, however, is an insufficient description of frosting: Intermediate spreading modes emerge due to collective effects that account for

\footnotetext{
*kondic@njit.edu

†vollmerd@mpip-mainz.mpg.de
}

Published by the American Physical Society under the terms of the Creative Commons Attribution 4.0 International license. Further distribution of this work must maintain attribution to the author(s) and the published article's title, journal citation, and DOI. Open access publication funded by the Max Planck Society. interactions between multiple droplets in two dimensions. In this case, regions of interconnected droplets and dry zones can occur simultaneously. Previously, this intermediate mode has been facilitated on chemically and geometrically patterned surfaces $[14,15]$. Here, we explore the patterns of frost, grown under the full spectrum of spreading modes, selected by modifying the amount of available condensed water and varying the set-point temperature $\left(-20\right.$ to $\left.-45^{\circ} \mathrm{C}\right)$, which affects frost patch nucleus formation. To control the droplet distribution, we use microstructured surfaces as a frost condenser. An infused fluorophore dyed silicone oil helps to increase the
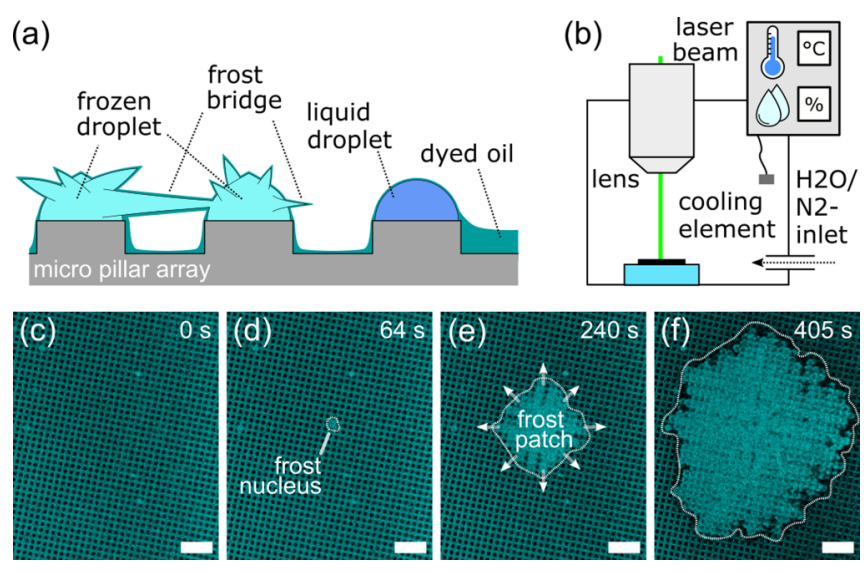

FIG. 1. (a) Cross-sectional schematic of frost spreading on micropillar array. Droplets sit on top of pillars. Frost bridges grow from frozen droplets toward liquid ones. Silicone oil is distributed in between the pillars and cloaks the droplets. (b) Experimental setup. (c)-(f) Frost formation and spreading: (c) Surface at $t=0 \mathrm{~s}$. Silicone oil in between the microstructure is cyan whereas the micropillar tops are black. (d) After some time ( $t=64 \mathrm{~s})$, one droplet starts freezing (i.e., frost patch nucleates). (e) and (f) Frost patch grows outwards by successively connecting neighboring droplets with frost bridges. Here, $\mathrm{RH}=34 \%, T_{\mathrm{set}}=-35^{\circ} \mathrm{C}$; scale bar is $200 \mu \mathrm{m}$. Temperatures on the surface were measured to be $3 \mathrm{~K}$ higher than $T_{\text {set }}$ 
contrast between surface and frost patterns which were observed via laser-induced fluorescence (LIF) microscopy. This imagining technique provides a large field of view $(2.5 \times$ magnification) and appropriate time resolution for transient frost pattern formation. Silicone oil cloaks water regardless of its aggregate state, which increases the visibility of frost but also affects frost spreading by slowing down the interdroplet vapor transport. We estimate the magnitude of vapor transport retardation by developing a resistance model and discuss it in the context of frost spreading. Furthermore, we find that patterns formed in intermediate spreading modes show fractal structure [16-18], therefore, slowing down the coverage speed of the frost [11]. Different topographies are formed, depending on the amount of available atmospheric water. The number and size of frost patches can be tuned by surface temperature.

\section{SURFACE PREPARATION AND EXPERIMENTAL SETUP}

Frost condenser surfaces were fabricated as follows: $170 \mu \mathrm{m}$ thick glass slides were coated with a rigid microstructure (SU-8) by photolithography. The microstructure is characterized by circular pillars (diameter: $30 \mu \mathrm{m}$, height: 10 and $20 \mu \mathrm{m})$ in a square lattice configuration $(30 \mu \mathrm{m}$ edge-to-edge distance). Fluorophore powder (Lumogen Red F300, BASF) was dissolved in silicone oil (polydimethylsiloxane $200 \mathrm{cSt}$, Gelest). The dyed oil was infiltrated into the microstructure. Silicone oil cloaks condensed water with an approximately $\delta \approx 10^{-8} \mathrm{~m}$ thick layer $[19,20]$ and creeps into the spreading frost patch [21]. The diffusive mobility of water in silicone oil $\left(D=2 \times 10^{-9} \mathrm{~m}^{2} / \mathrm{s}\right.$ [22]) allows water molecules to easily pass through the oil [23]. Hence, the silicone oil does not insulate water vapor transport but rather constitutes a resistance towards the diffusing water molecules. However, it helps to visualize the frost patches by increasing the contrast on the surface. The impregnated microstructure is placed on a Peltier element which cools the surface down to controllable subzero temperatures $T_{\text {set }}$. To form frost, the cooled surface is sealed in an environmental chamber. Nitrogen gas with preset water vapor content [i.e., relative humidity $(\mathrm{RH})]$ is introduced into the chamber for $30 \mathrm{~s}$. Thereafter, the system relaxes to a steady state. All processes (i.e., frost formation and spreading) on the surface were monitored via LIF microscopy operated with a $2.5 \times$ objective lens (dry; Leica HC PL FLUOTAR $2.5 \times / 0.07)$, Fig. 1(b).

\section{FROST FORMATION AND SPREADING}

Right after humidified nitrogen gas is introduced into the sealed chamber, supercooled droplets condense on the micropillar tops, Fig. 1(c). Condensation of droplets is accompanied by falling relative humidity in the chamber from supersaturation to saturation with respect to the cooled surface [23]. Supercooled droplets remain liquid for some time before they randomly start to freeze. Freezing of a droplet becomes visible via LIF due to accumulation of the lubricant leading to change in contrast, Fig. 1(d). We identify a single frozen droplet as the nucleus of a frost patch. The frost patch grows as a continuous domain from the nucleus outwards, Fig. 1(e). The growth of the domain is facilitated by the vapor flux,
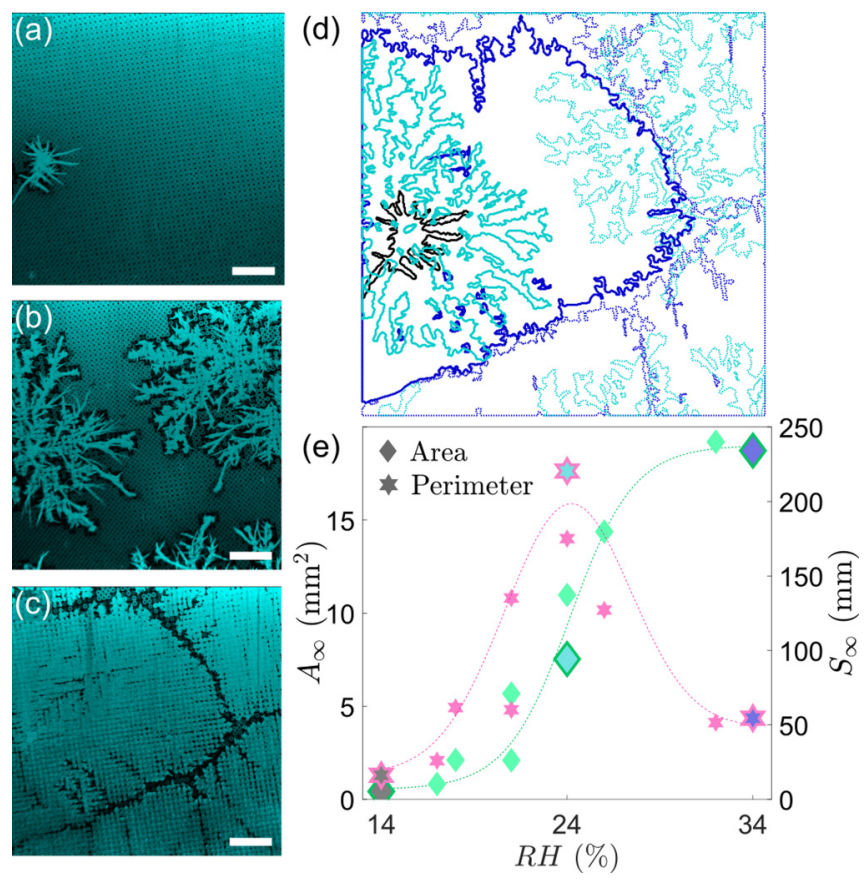

FIG. 2. Frost patterns for different RHs and $T_{\text {set }}=-30{ }^{\circ} \mathrm{C}$ at the steady state. (a) $\mathrm{RH}=14 \%$. A single frost patch with arbitrarily directed frost spikes. (b) $\mathrm{RH}=24 \%$. Multiple frost patches form. (c) $\mathrm{RH}=34 \%$. The surface is nearly entirely covered by frost. Scale bars are $500 \mu \mathrm{m}$. (d) Boundary contour of frost patches, $\mathrm{RH}=14 \%$ (black), $\mathrm{RH}=24 \%$ (turquoise), and $\mathrm{RH}=34 \%$ (blue) overlaid. For all three RH configurations, frost patches (solid thick lines) were triggered from the same defect. Other neighboring frost patches are outlined with faint lines. (e) Area $A_{\infty}$, (green diamonds), and perimeter $S_{\infty}$, (pink stars) versus RH. Filled symbols at 14\%, 24\%, and $34 \% \mathrm{RH}$ correspond to patterns in (d). The area of frost coverage increases monotonously with peak relative humidity, whereas the perimeter passes a maximum around $\mathrm{RH}=24 \%$. The dotted line serves as a guide to the eye.

coming from liquid droplets. In Sec. IV, we introduce a framework of this flux. While the frost patch grows, the region right in front of the frost patch becomes darker, making the perimeter more pronounced. This is caused by local oil depletion, i.e., oil is sucked into the dendritic frost patch [21,24], Fig. 1(f). The frosting process ends when all liquid water on the cooled surface becomes either solid as part of the frost patches or vapor as it reevaporates back into the atmosphere. As set-point temperature and RH are the expected governing system parameters, we varied those and discuss their respective impact, starting with RH.

\section{A. Frost spreading at differing humidity}

$\mathrm{RH}$ is determined by the water content in the nitrogen flux at an ambient temperature of $20 \pm 2{ }^{\circ} \mathrm{C}$. We discuss three characteristic configurations at which the relative humidity peaked at $14 \%, 24 \%$, and $34 \%$. Each configuration is observed in a steady state $\left(t \rightarrow t_{\infty}\right)$ and the field of observation $\left(3220 \times 3220 \mu \mathrm{m}^{2}\right)$ is kept on the same surface spot, Figs. 2(a)-2(c). For $\mathrm{RH}=14 \%$, only a single frost patch formed, Fig. 2(a) and Supplemental Material video 
[25]. While this patch starts forming frost bridges, neighboring droplets completely evaporate before the bridges reach them. The cascading process is interrupted. Still, water vapor from evaporating droplets slowly attaches on the single frost patch, forming randomly directed spiky tips. Increasing the water content to an intermediate amount $(\mathrm{RH}=24 \%)$ leads to the formation of three visible frost patches within the field of observation, Fig. 2(b) and Supplemental Material video [25]. Each frost patch grows larger, compared to the patch in drier atmosphere. During growth, frost bridges reach out for their neighboring liquid droplets to connect them. However, this does not always occur, as reflected by the dark regions between branches, which are not covered by frost. Instead, they evaporate entirely before frost bridges can reach them, leaving empty micropillar tops behind. The pattern shows branching arms, angled at approximately $45^{\circ}$ to the main arm. This roots from the anisotropy under which the frost patch grows, imposed by the microarray lattice. For a very humid atmosphere, the nucleated frost patches connect nearly all liquid droplets on the surface, Fig. 2(c) and Supplemental Material video [25]. Growing frost patches halt when they approach each other, separated by a "ditch." Overlaying the boundary contours of the frost patch patterns, grown by the three RH configurations, unravels descriptively how different amounts of water content in the atmosphere lead to different patterns and how they are related, Fig. 2(d). Frost at RH = $14 \%$ nucleated from a surface defect. The same surface defect triggered frost for the other two RH configurations. The size of the regions uncovered by frost gradually decreases with increasing atmospheric humidity. While frost covered area $A_{\infty}$ increases monotonously (green diamonds) with relative humidity, the cumulative perimeter $S_{\infty}$ (pink stars) passes a maximum, Fig. 2(e). This indicates that increased atmospheric humidity not only leads to more surface coverage (increased $A_{\infty}$ ), but also to smoother frost patch perimeters (decreased $S_{\infty}$ ). The minimum of $S_{\infty}$ and $A_{\infty}$ at RH $=14 \%$ stems from the solely diffusive driven growth mode which is fundamentally different from the other two cases. Droplets do not connect, leading to a small frost patch with small $S_{\infty}$ and $A_{\infty}$.

\section{B. Dynamic tracking of spreading frost patches}

To understand how different patterns emerge, we track the evolution of the covered area $A(t)$ and perimeter $S(t)$ of the frost patches during their formation and growth for all three RH configurations, Figs. 3(a) and 3(b). The field of observation was increased to $5031 \times 1962 \mu \mathrm{m}^{2}$ to obtain better sampling statistics. The dynamically acquired data set was processed via outlier filtering, Appendix A. Data points with poor signal to noise ratio in the early stage of the experiments $(t<60 \mathrm{~s})$ were excluded. To validate the dynamic measurements, we conducted measurements on surfaces with enhanced frost detection and a larger field of view after reaching a steady state $\left(t \rightarrow t_{\infty}\right)$, Table I. Note that the trends of $A_{\infty}$ and $S_{\infty}$ are consistent between dynamic [Figs. 3(a) and 3(b)] and static measurements (Table I). Normalizing the recorded data by $A_{\infty}$ shows that $A(t)$ evolves for all three cases as a stretched S-shaped curve, Fig. 3(c). The normalized plot reveals that area growth is fastest when the humidity
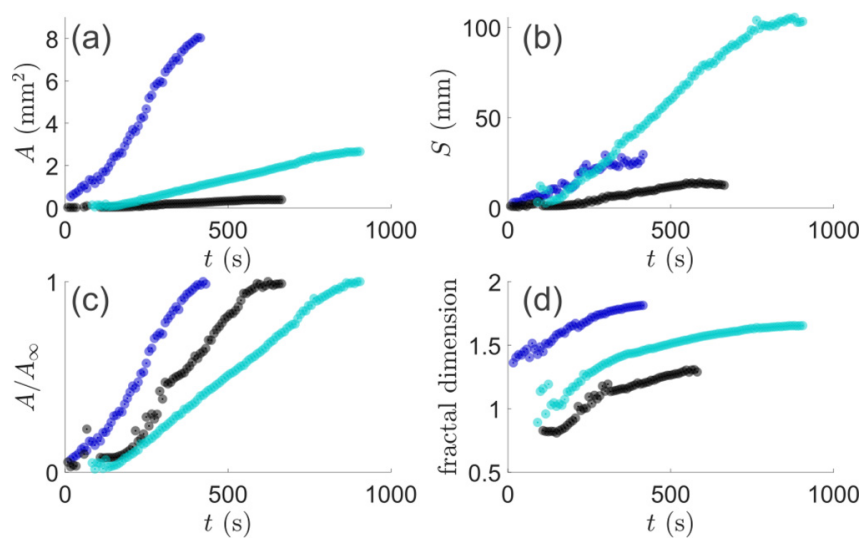

FIG. 3. Evolution of frost patch over time for $\mathrm{RH}=14 \%$ (black), $\mathrm{RH}=24 \%$ (turquoise), and $\mathrm{RH}=34 \%$ (blue). (a) Covered area $A(t)$; (b) perimeter $S(t)$; (c) normalized area $A(t) / A_{\infty}$; and (d) fractal dimension $d_{f}$.

is at its highest (blue curve), followed by the lowest $\mathrm{RH}$ configurations (black curve). This is reflected in the average frost front speed, which is $1.4 \pm 0.7 \mu \mathrm{m} / \mathrm{s}$ for the highest and $0.2 \pm 0.1 \mu \mathrm{m} / \mathrm{s}$ for the lowest humidity, Appendix B. Even though growth times for $\mathrm{RH}=14 \%\left(t_{\infty}=720 \mathrm{~s}\right)$ and $\mathrm{RH}=34 \%\left(t_{\infty}=540 \mathrm{~s}\right)$ are comparable, the former grows much slower, which stems from a fundamentally different growth mode. For $\mathrm{RH}=14 \%$, the frost patch grows mainly by forming spiky tips. These tips are only able to grow as long as the gaseous ambient is supersaturated with respect to the surface (or the nucleus). This condition holds as long as condensing droplets evaporate back into the atmosphere. The evaporation of the droplets constitutes the time-limiting factor. For $R H=34 \%, t_{\infty}$ is governed by successively connected droplets via ice bridges, which is a diffusion-type process. As freezing of connected droplets is fast compared to the growth of frost bridges, the diffusion process is sped up as the droplet size increases [7]. Interestingly, the intermediate RH configuration appears to exhibit the longest growth time (turquoise curve) with an average frost front speed of $0.08 \pm 0.04 \mu \mathrm{m} / \mathrm{s}$. Droplets do connect via frost bridges, however, this process is occasionally interrupted by the premature droplet evaporation before frost bridges could reach them. These "dead ends" throttle the growth. Considering frost spreading at $\mathrm{RH}=$ $24 \%$, it becomes evident that frost does not only spread per: (i) successful interdroplet bridge connection (as for $\mathrm{RH}=$ $34 \%$ ) or (ii) disjoint, diffusive growth (as for $\mathrm{RH}=14 \%$ ). In between the two extreme scenarios fractal-like branching

TABLE I. Steady state area $A_{\infty}$ and perimeter $S_{\infty}$ for dry, intermediate, and humid RH configurations. $t_{\infty}$ is the total time taken for the steady state to be reached. Data correspond to steady state patterns with high precision extraction. Plane of $6440 \times 3220 \mu \mathrm{m}^{2}$.

\begin{tabular}{lcccc}
\hline \hline $\mathrm{RH}(\%)$ & $A_{\infty}\left(\mathrm{mm}^{2}\right)$ & $S_{\infty}(\mathrm{mm})$ & $t_{\infty}(\mathrm{s})$ & $d_{f, \infty}{ }^{\mathrm{a}}$ \\
\hline 14 & 0.5 & 16 & 720 & 1.3 \\
24 & 7.5 & 220 & 940 & 1.7 \\
34 & 18.7 & 55 & 540 & 1.9 \\
\hline \hline
\end{tabular}

${ }^{\mathrm{a}}$ Obtained by box counting [26]. 
arms develop, generating frost patterns of variable surface coverage. The spreading mode can, therefore, be tuned by the ambient humidity.

Figure 3(d) shows the fractal dimension $d_{f}$ (computed using the box-counting method [26]) of the growing patches. For all three configurations, $d_{f}$ grows monotonously and converges to differing steady state values for the respective humidity configurations. We measured the fractal dimension for steady states $d_{f, \infty}$ again with enhanced frost detection, Table I. For $R=34 \%, d_{f, \infty} \approx 1.9$, which is characteristic for correlated aggregation processes where the fractal dimension is slightly lower than the ordinary Euclidean dimension (i.e., 2) [27]. This result can be understood considering that all condensed droplet connect and contribute to the frost patch. For $\mathrm{RH}=24 \%, d_{f, \infty} \approx 1.7$. This value characterizes diffusion limited aggregates [16]. This is in line with the observation that not all droplets are successively connected, but some spots are left dry. Finally, for the driest scenario $(\mathrm{RH}=14 \%) d_{f, \infty} \approx 1.3$. This value does not correspond to either correlated or diffusive limited aggregation. This supports the interpretation for $\mathrm{RH}=14 \%$ being a different mode of growth.

\section{Frost spreading at differing temperature}

To understand the influence of temperature, we varied $T_{\text {set }}$ within $-20{ }^{\circ} \mathrm{C}$ and $-45^{\circ} \mathrm{C}$. One consequence of decreasing the set-point temperature is that supersaturation of ambient water vapor with respect to the cooled surface is stronger. This yields more water condensate on the surface. In this sense, varying any of the control parameters either $T_{\text {set }}$ or RH leads to variations in the amount of liquid condensate on the surface. This affects frost pattern growth, similar to observations in the preceding sections. In order to minimize the effect of $T_{\text {set }}$ on the amount of feed condensate and frost patterns, we choose a constant humidity peak at $\mathrm{RH}=28 \pm 1 \%$ for all $T_{\text {set }}$ 's in the experiments discussed next. For this humidity configuration, the patterns' perimeter grows relatively smoothly, cf. Fig. 2(e). The patterns grow in correlated mode [cf. Fig. 2(c)] for all set-temperature configurations and, therefore, pattern-fractality variations are suppressed. The steady state frost pattern formed at $T_{\text {set }}=-25^{\circ} \mathrm{C}$ is characterized by few large frost patches, Fig. 4(a). Each patch developed branching arms separated by unconnected areas (fine dark boundaries) within each patch. Decreasing the temperature to $T_{\text {set }}=-35^{\circ} \mathrm{C}$ results in comparatively more but smaller frost patches, Fig. 4(b). For $T_{\text {set }}=-45^{\circ} \mathrm{C}$ this trend almost reaches the theoretical limit of one drop per pillar, Fig. 4(c). At this limiting condition, condensing droplets do not freeze in a staggered manner but simultaneously right after condensation. This creates almost as many frost patches as the number of micropillars on the surface. We observe that steady states are reached faster (i.e., smaller $t_{\infty}$ ) for decreasing $T_{\text {set }}$, Fig. 4(d). The reason for smaller $t_{\infty}$ lies in the increased number of frost patch nuclei per area $n$, which we count as soon as they emerge, Fig. 4(e). The highest possible number of frost patch nuclei is limited by the micropillar density of the surface, which is demarcated by the dashed line. Plotting the counted $n$ against the $T_{\text {set }}$ indicates an Arrhenius behavior of the frost (d)

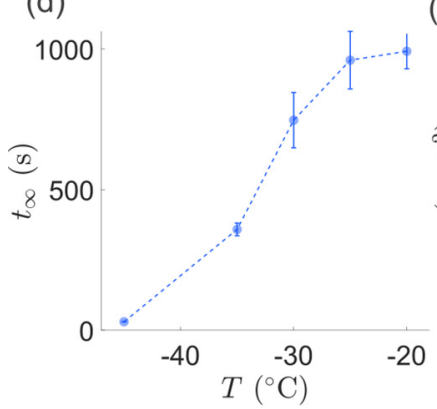

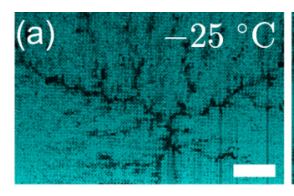

(e)
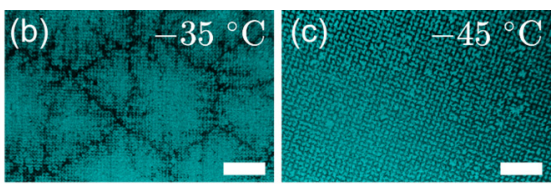

$10^{-3}$

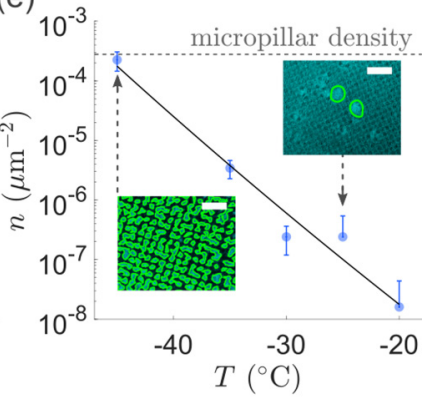

FIG. 4. Steady state frost pattern for $\mathrm{RH}=28 \pm 1 \%$. Set point temperature $T_{\text {set }}$ is (a) $-25^{\circ} \mathrm{C}$, (b) $-35^{\circ} \mathrm{C}$, and (c) $-45^{\circ} \mathrm{C}$. Scale bars are $500 \mu \mathrm{m}$. The dependence of the relaxation time (d) $t_{\infty}$ and of the number of nuclei per area (e) $n$ on imposed temperature $T_{\text {set }}$. The insets show that fewer nuclei formed at $T_{\text {set }}=-25^{\circ} \mathrm{C}$ and many nuclei formed at $T_{\text {set }}=-45^{\circ} \mathrm{C}$ set point temperature. Nuclei boundaries are emphasized in green. The dashed line depicts micropillar density (upper nucleus limit), the solid line shows an exponential fit shown in Eq. (1). The scale bar in insets is $200 \mu \mathrm{m}$.

patch nucleation,

$$
n \sim \exp \left[-g\left(T_{\text {set }}\right) / k_{B} T\right],
$$

as depicted by the black solid line in the plot. Here, $k_{B}$ is the Boltzmann constant, and $T$ is the droplets' temperature. Frost nucleus formation is linked to overcoming the energetic barrier $g\left(T_{\text {set }}\right) \approx 10 k_{B} T$ (i.e., freezing). The value for $g\left(T_{\text {set }}\right)$ is obtained by fitting Eq. (1) to the counted $n$. The black line in Fig. 4(e) corresponds to the fit. Note, that the trend of $n$ indicates a vanishing nucleation barrier for lower $T_{\text {set }}$.

\section{WATER VAPOR TRANSPORT IN A CLOAKED DROPLET ARRAY}

Spreading of the frost domain is facilitated by the growth of frost bridges, emerging from the nucleus. Here we briefly discuss a framework for the governing vapor transport, which provides a basic rational that is useful for understanding the experimental results. The saturation concentration of water around each droplet is different, depending on its aggregate state [28]. This roots from differing chemical potentials of liquid and frozen water at equal temperature. If a frozen droplet neighbours a liquid droplet, the water concentration increases from the frozen to the liquid drop. Therefore, diffusive water transport is facilitated from the liquid to the solid droplet, Fig. 5(a). Water molecules attach on the frozen surface $S$ at a rate of

$$
J=D \nabla c \cdot \boldsymbol{n},
$$

where $\nabla c$ is the gradient of the water concentration on the nucleus' surface $S$ and $\boldsymbol{n}=\boldsymbol{\nabla} S /\|\nabla S\|$ is the surface normal vector. Although droplets are three-dimensional objects, the mass flow on their surface per Eq. (2) acts dominantly from 
(a)

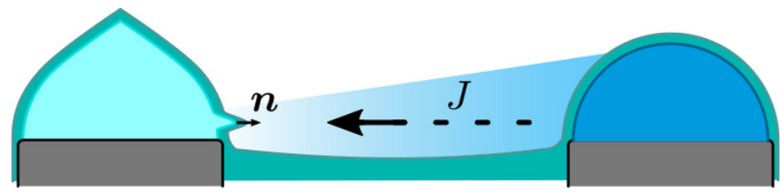

(b)

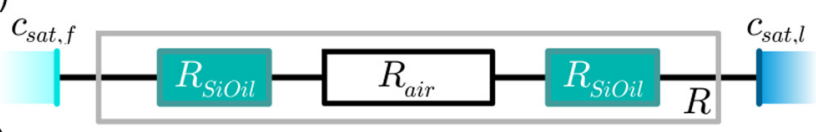

(c)

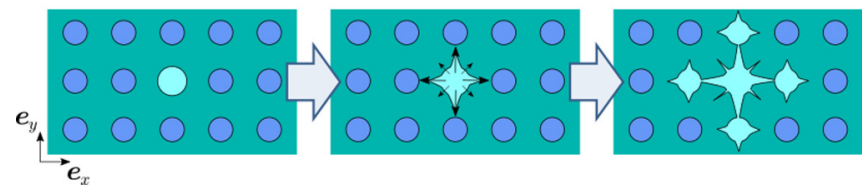

FIG. 5. Diffusive flux from liquid to frozen droplet. (a) Schematics of water vapor gradient from liquid to solid droplet. Water attaches on the solid droplet, forming an emerging frost bridge. Both the liquid and the solid droplet are cloaked by a thin silicone oil layer. (b) Serial flux resistance. Water molecules are saturated on the solid $\left(c_{\text {sat }, s}\right)$ and on the liquid $\left(c_{\text {sat }, l}\right)$ droplet interface towards silicone oil. Water travels from the liquid droplet trough the first silicone oil layer $\left(R_{\mathrm{Si} \text { oil }}\right)$, then through air $\left(R_{\mathrm{air}}\right)$ and then, again, through a silicone oil layer $\left(R_{\mathrm{Si} \text { oil }}\right)$ before it finally attaches on the solid droplet.

droplet to droplet on the plane of the substrate. We assume that the vapor transport is in quasiequilibrium, yielding a divergence free flux field, i.e., $\nabla \cdot J=0$. Droplets are cloaked by a thin layer of silicone oil, independent of their aggregate state (liquid or frozen) $[19,20]$. Water is soluble in silicone oil [22], which leads to migration of water molecules from the droplets into the silicone oil layer. Directly at the shared interface between water and silicone oil, the water molecules are in local equilibrium. This implies that here, silicone oil is saturated with water. In the steady state, the mass flux from liquid to frozen droplet scales as

$$
J=\left(c_{\text {sat }, s}-c_{\text {sat }, l}\right) / R,
$$

where $c_{\mathrm{sat}, l}$ and $c_{\mathrm{sat}, s}$ are the saturation concentration of water around a liquid and a frozen droplet, respectively. $R$ denotes the flux resistance which we discuss next. While water molecules migrate through silicone oil and through air, they experience in each phase a different diffusive resistance, i.e., $R_{\text {Si oil }}$ and $R_{\text {air }}$, respectively. Those resistances appear in series, Fig. 5(b). Hence, they add up to a total resistance as

$$
R=R_{\text {Si oil }}+R_{\text {air }}+R_{\text {Si oil }}=\frac{\delta_{\text {air }}}{D_{\text {air }}}+2 \frac{\delta}{D} .
$$

Here, the diffusivity of water vapor in air is $D_{\text {air }}=3 \times$ $10^{-5} \mathrm{~m}^{2} / \mathrm{s}$ [29]. The distance, separating droplets in air is here denoted with $\delta_{\text {air }}$. Considering that $i=1,2, \ldots, m$ droplets are distributed on the surface, we can evaluate the flux $j_{i j}$ at position $\boldsymbol{x}_{i}$, coming from a droplet at position $\boldsymbol{x}_{j}$ per

$$
j_{i j}=\frac{c_{i}-c_{j}}{\frac{\delta_{i j}}{D_{\mathrm{air}}}+2 \frac{\delta}{D}}
$$

This expression for the flux does not only account for the fluxes between a liquid and a frozen droplet but between any two arbitrary droplets $i, j$. The resistance will then depend

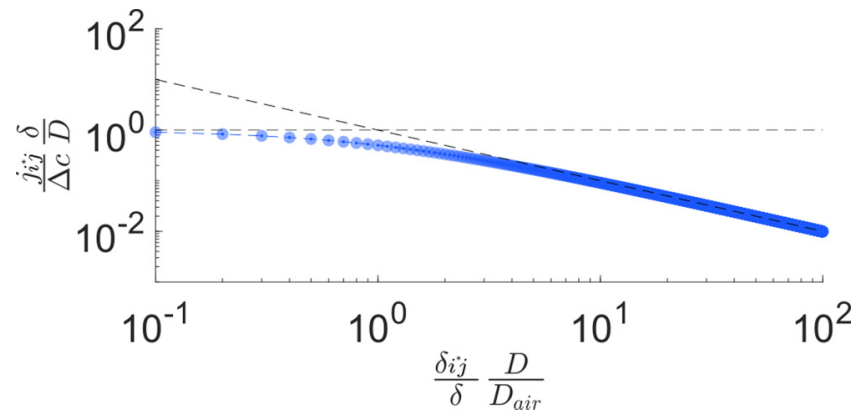

FIG. 6. Nondimensional vapor flux as a function of nondimensional separation distance (here, the distance between pillars is 0.1 ; each circular symbol corresponds to a pillar site). Individual flux components $j_{i^{*} j}$. Regime of oil limited transport (horizontal dashed line) crosses over into air limited transport (inclined dashed line). The respective dashed lines are plotted by using the discussed flux expression $j_{i^{*} j}$ with only one of the two resistance components.

on the separation distance between these $i, j$ droplets, which computes as $\delta_{i j}=\left\|\boldsymbol{x}_{i}-\boldsymbol{x}_{j}\right\|$. The concentrations $c_{i}, c_{j}$ are determined by the aggregate state of the $i, j$ th droplets, respectively, and are assumed to correspond to the saturation vapor concentration of either liquid or solid water, namely,

$$
c_{i}=\left\{\begin{array}{cl}
c_{\mathrm{sat}, s} & \text { if the droplet is frozen, } \\
c_{\mathrm{sat}, l} & \text { if the droplet is liquid. }
\end{array}\right.
$$

In order to determine the total flux to a droplet at $\boldsymbol{x}_{i}$, Eq. (5) has to be summed up over all other droplets $j$ as

$$
J_{i}=\sum_{j, j \neq i}^{m} j_{i j} .
$$

Frost bridges gradually accumulate mass per Eq. (2) and grow towards the neighboring liquid droplets. When the frost bridge connects with a neighboring liquid droplet, the droplet freezes immediately. At the freshly frozen droplet, new frost bridges grow toward their neighboring liquid droplets, Fig. 5(c). This cascading process leads to growth of a continuous frost domain.

The flux on a frozen droplet from gradually further separated liquid ones is plotted in Fig. 6(a). Here, we consider a frozen droplet at fixed $i=i^{*}$. In the plot, the flux $j_{i^{*} j}$ is nondimensionalized with the flux magnitude within the silicone oil, i.e., $\delta /(\Delta c D)$ where $\Delta c=c_{\mathrm{sat}, s}-c_{\mathrm{sat}, l}$. To nondimensionalized the separation distance $\delta_{i^{*} j}$, we choose the scale $D /\left(\delta D_{\text {air }}\right)$. In the immediate vicinity of the frozen droplet, the fluxes are strong and become gradually weaker as further away the liquid droplets are located. At a certain distance, the flux becomes drastically weaker with increasing separation distance. In our system, this crossover occurs at approximately $600 \mu \mathrm{m}$ from the frozen droplet. This crossover can be explained by revisiting Eq. (5): We note that at $\delta_{i * j} \approx 600 \mu \mathrm{m}$ both terms in the denominator become comparable. This implies that fluxes in the direct vicinity of a frozen droplet are limited by the resistance that water molecules experience in silicone oil. We hypothesize that the interplay between fluxes per Eq. (7) and the amount of condensed water will determine the outcome of frost spreading. This estimate of governing flux resistance is only valid for fluxes from liquid droplets to 
(a)

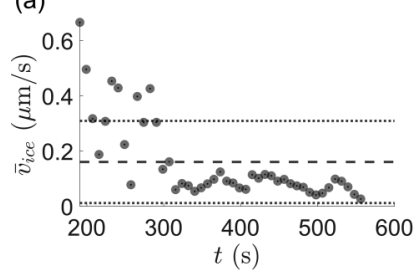

(b)

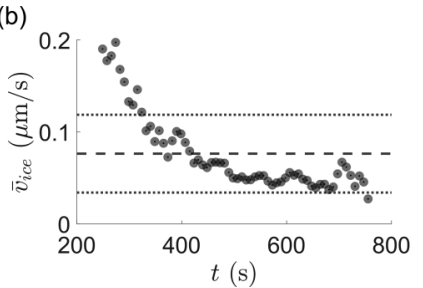

(c)

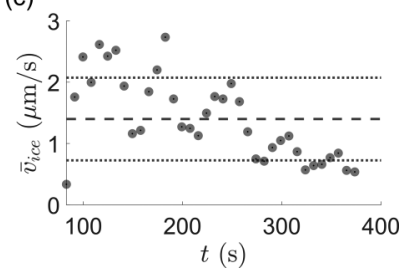

FIG. 7. Average frost front propagation speed for (a) $\mathrm{RH}=14 \%$, (b) $\mathrm{RH}=24 \%$, and (c) $\mathrm{RH}=34 \%$. The dashed line corresponds to the time-averaged speed which is (a) $0.2 \pm 0.1 \mu \mathrm{m} / \mathrm{s}$, (b) $0.08 \pm 0.04 \mu \mathrm{m} / \mathrm{s}$, and (c) $1.4 \pm 0.7 \mu \mathrm{m} / \mathrm{s}$. The dotted lines demarcate the $1-\sigma$ (standard deviation) band.

a single frozen one. On larger scales, however, this criterion is not necessarily fulfilled anymore. We expect that the picture becomes more complicated, once frost patches are formed and spread on the surface.

\section{CONCLUSION}

To summarize, tuning the relative humidity produces different modes of spreading; in between the two cases of droplets fully interconnected by frost bridges (leading to almost complete surface coverage) and pure diffusion driven growth (leading to spiky-looking objects covering a small part of the domain), we find a mixture of both modes, which exhibits fractal-like properties. Furthermore, the set-point temperature is a direct proxy for the frost patch nucleus formation which is limited by the energy barrier. We note that the formation of branching arms appears to be inherently encoded in the condensation-frosting process. The weaker manifestation of the branching arms for lower set-point temperatures is, hence, rather a consequence of a faster approach to steady state caused by increased number of nucleating sites. We hope that our results will inspire further experimental and theoretical work that will lead to a comprehensive understanding of pattern formation occurring during frosting.

\section{ACKNOWLEDGMENTS}

This work was supported by the German Research Foundation (DFG) with the Priority Programme 2171 (L.H., D.V.), the European Union's Horizon 2020 Research and Innovation Program LubISS No. 722497 (W.S.Y.W. and D.V.), the Max Planck-University Twente Center for Complex Fluid Dynamics (D.V.), U.S. National Science Foundation Grants No. NSF CBET-1604351 and No. DMS-1815613, and NJIT Faculty Seed Grant (L.K.). We thank H.-J. Butt, A. Naga, and P. Stephan for stimulating discussions.

\section{APPENDIX A: RUNNING MEDIAN OUTLIER FILTER}

The running median outlier filter is excluding data points which exceed three scaled median absolute deviations (sMAD) within their direct environment. We used the the
MATLAB function isoutlier() for this purpose [30]. The running median $\varphi_{i}^{m}$ in a set $\left[\phi_{i}\right]$ defines the median of $m$ points at a centric position $i$. The running median in an environment of, e.g., $m=10$ points reads

$$
\varphi_{i}^{10}=\operatorname{median}\left(\phi_{j}\right)
$$

with $j=[i-5, i+5]$. sMAD is defined as

$$
\operatorname{sMAD}_{i}=c \operatorname{median}\left(\left|\phi_{j}-\varphi_{i}^{10}\right|\right),
$$

with $c=-1 /[\sqrt{2} \operatorname{erfcinv}(3 / 2)]$. Hence, we find

$$
\phi_{i}= \begin{cases}\phi_{i} & \text { for } \phi_{i} \leqslant 3 \mathrm{sMAD} \\ \varnothing & \text { for } \phi_{i}>3 \mathrm{sMAD}\end{cases}
$$

\section{APPENDIX B: AVERAGE FROST FRONT PROPAGATION SPEED}

In order to compute the average frost front propagation speed, we consider how the area $A(t)$ grows by means of an expanding perimeter $S(t)$ with a velocity $\boldsymbol{v}_{\text {ice }}$ at the perimeter,

$$
\frac{d A}{d t}=\dot{A}=\oint\left(\boldsymbol{n} \cdot \boldsymbol{v}_{\text {ice }}\right) d S .
$$

Here, $\boldsymbol{n}=\nabla S /\|\nabla S\|$ is the perimeter's normal vector. We spatially average the velocity, normal to the perimeter over the perimeter yielding

$$
\bar{v}_{\text {ice }}=\frac{\oint\left(\boldsymbol{n} \cdot \boldsymbol{v}_{\text {ice }}\right) d S}{\oint d S} .
$$

Since $\oint d S=S(t)$, Eqs. (B1) and (B2) give

$$
\bar{v}_{\text {ice }}=\frac{\dot{A}}{S} \text {. }
$$

We compute $\dot{A}$ from the measurements, cf. Fig. 3(a). In order to make the derivative estimate of each data point more robust, we utilize four neighboring points (two preceding, two succeeding) and compute the linear regression. The derivative is taken as the linear regression's slope. Figure 7 shows $\bar{v}_{\text {ice }}$ for the data in Figs. 3(a) and 3(b). Finally, we compute the time mean and its standard derivation of $\bar{v}_{\text {ice }}$ during the main phase (100-800 s) of the frost growth.
[1] H. Zhao and D. Beysens, From droplet growth to film growth on a heterogeneous surface: Condensation asso- ciated with a wettability gradient, Langmuir 11, 627 (1995). 
[2] D. Beysens, Dew nucleation and growth, C. R. Phys. 7, 1082 (2006).

[3] S. Anand, K. Rykaczewski, S. B. Subramanyam, D. Beysens, and K. K. Varanasi, How droplets nucleate and grow on liquids and liquid impregnated surfaces, Soft Matter 11, 69 (2015).

[4] B. Na and R. L. Webb, A fundamental understanding of factors affecting frost nucleation, Int. J. Heat Mass Transf. 46, 3797 (2003).

[5] A. G. Marín, O. R. Enríquez, P. Brunet, P. Colinet, and J. H. Snoeijer, Universality of Tip Singularity Formation in Freezing Water Drops, Phys. Rev. Lett. 113, 054301 (2014).

[6] K. G. Libbrecht, Physical Dynamics of Ice Crystal Growth, Annu. Rev. Mater. Res. 47, 271 (2017).

[7] J. Guadarrama-Cetina, A. Mongruel, W. González-Viñas, and D. Beysens, Percolation-induced frost formation, Europhys. Lett. 101, 16009 (2013).

[8] S. Nath, S. F. Ahmadi, and J. B. Boreyko, A review of condensation frosting, Nanoscale Microscale Thermophys. Eng. 21, 81 (2017).

[9] J. B. Boreyko and C. P. Collier, Delayed frost growth on jumping-drop superhydrophobic surfaces, ACS Nano 7, 1618 (2013).

[10] H. R. Pruppacher and J. D. Klett, Microphysics of Clouds and Precipitation, 2nd rev. ed. (Kluwer Academic Publishers, New York, 2004), pp. 546-550.

[11] J. Petit and E. Bonaccurso, General frost growth mechanism on solid substrates with different stiffness, Langmuir 30, 1160 (2014).

[12] S. Nath and J. B. Boreyko, On localized vapor pressure gradients governing condensation and frost phenomena, Langmuir 32, 8350 (2016).

[13] S. Nath, S. F. Ahmadi, and J. B. Boreyko, How ice bridges the gap, Soft Matter 16, 1156 (2020).

[14] S. F. Ahmadi, S. Nath, G. J. Iliff, B. R. Srijanto, C. P. Collier, P. Yue, and J. B. Boreyko, Passive antifrosting surfaces using microscopic ice patterns, ACS Appl. Mater. Interfaces 10, 32874 (2018).

[15] Y. Yao, T. Y. Zhao, C. Machado, E. Feldman, N. A. Patankar, and K. C. Park, Frost-free zone on macrotextured surfaces, Proc. Natl. Acad. Sci. U.S.A. 117, 6323 (2020).
[16] T. A. Witten and L. M. Sander, Diffusion-Limited Aggregation, A Kinetic Critical Phenomenon, Phys. Rev. Lett. 47, 1400 (1981).

[17] B. Mandelbrot, How long is the coast of Britain? Statistical selfsimilarity and fractional dimension, Science 156, 636 (1967).

[18] E. Ben-Jacob, From snowflake formation to growth of bacterial colonies, Contemp. Phys. 34, 247 (1993).

[19] V. Bergeron and D. Langevin, Monolayer Spreading of Polydimethylsiloxane Oil on Surfactant Solutions, Phys. Rev. Lett. 76, 3152 (1996).

[20] D. Daniel, J. V. Timonen, R. Li, S. J. Velling, and J. Aizenberg, Oleoplaning droplets on lubricated surfaces, Nat. Phys. 13, 1020 (2017).

[21] W. S. Y. Wong, K. I. Hegner, V. Donadei, L. Hauer, A. Naga, and D. Vollmer, Capillary balancing: Designing frost-resistant lubricant-infused surfaces, Nano Lett. 20, 8508 (2020).

[22] Y. Tamai, H. Tanaka, and K. Nakanishi, Molecular simulation of permeation of small penetrants through membranes. 1. Diffusion coefficients, Macromolecules 27, 4498 (1994).

[23] L. Hauer, W. S. Wong, V. Donadei, K. I. Hegner, L. Kondic, and D. Vollmer, How frost forms and grows on lubricated microand nanostructured surfaces, ACS Nano 15, 4658 (2021).

[24] K. E. Witt, S. F. Ahmadi, and J. B. Boreyko, Ice wicking, Phys. Rev. Fluids 4, 024002 (2019).

[25] See Supplemental Material at http://link.aps.org/supplemental/ 10.1103/PhysRevE.104.044901 for movie of frost formation and spreading at $(\mathrm{RH}=14 \%, 24 \%, 34 \%)$ and $T_{\text {set }}=-35^{\circ} \mathrm{C}$.

[26] L. S. Liebovitch and T. Toth, A fast algorithm to determine fractal dimensions by box counting, Phys. Lett. A 141, 386 (1989).

[27] M. J. Vold, Computer simulation of floc formation in a colloidal suspension, J. Colloid Sci. 18, 684 (1963).

[28] D. M. Murphy and T. Koop, Review of the vapour pressures of ice and supercooled water for atmospheric applications, Q. J. R. Meteorol. Soc. 131, 1539 (2005).

[29] E. L. Cussler, Diffusion: Mass Transfer in Fluid Systems, 3rd ed. (Cambridge University Press, Cambridge, UK, 2009), p. 118.

[30] MATLAB, version 9.9.0.1467703 (R2020b) (The MathWorks, Inc., Natick, MA, 2020) https://uk.mathworks.com/help/ matlab/ref/isoutlier.html. 